\title{
Genetic polymorphism of DNATyper X19 kit in Northern Chinese Han people
}

\author{
YU Zhengliang ${ }^{1}$, CAI Huiju', MA Wenhua ${ }^{1}$, MO Xiaoting ${ }^{1}$, YAO Yiren ${ }^{1}$, WANG Le ${ }^{1}$, LIU Chao ${ }^{2}$, \\ ZHAO Xingchun ${ }^{1}$, LI Wanshui ${ }^{1, *}$
}

${ }^{1}$ Institute of Forensic Science, Ministry of Public Security, Beijing 100038, China.

${ }^{2}$ Guangzhou Forensic Institute, Guangzhou 510000, China.

\begin{abstract}
How to cite this paper: YU Zhengliang, CAI Huiju, MA Wenhua, MO Xiaoting, YAO Yiren, WANG Le, LIU Chao, ZHAO Xingchun, LI Wanshui. (2020) Genetic polymorphism of DNATyper X19 kit in Northern Chinese Han people. International Journal of Clinical and Experimental Medicine Research, 4(2), 28-33.

DOI: $10.26855 /$ ijcemr.2020.04.005
\end{abstract}

Received: March 22, 2020

Accepted: April 28, 2020

Published: May 7, 2020

*Corresponding author: LI Wanshu, Institute of Forensic Science, Ministry of Public Security, Beijing 100038, China.

Email: liwanshui68@sina.com

\begin{abstract}
Objective. To evaluate the value of DNATyper X19 kit in the forensic application. Methods. In this study, we carried out a survey of genetic polymorphism from 374 Northern Chinese Han individuals. Results. A total of 140 alleles were detected among the 18 X-STR loci from 374 Northern Chinese Han individuals, 228 haplotypes were detected from the 228 males, the diversity is $100 \%$. No markers departed from Hardy-Weinberg equilibrium after applying Bonferroni's correction for multiple testing $(\mathrm{p}=0.05 / 18)$, GATA31E08-DXS6797, DXS8377-DXS10079 were in linkage disequilibrium (LD) in this study $(\mathrm{p}=0.05 / 153)$. The polymorphism information content (PIC) was $0.434-0.908$ and the discrimination power (DP) was 0.432-0.983. The combined discrimination power was 0.99999993 for male and 0.999999992 for female. The combined mean exclusion chance was 0.999993 in duo cases (CMECduo) and 0.999999994 in trio case (CMECtrio). Conclusions. DNATyper X19 kit showed potential value for complicated paternity cases.
\end{abstract}

\section{Keywords}

Forensic Genetics, X Chromosome, X-STR

\section{Introduction}

Association with sex-linked genetic characteristics has made X-STR markers good candidates for efficient complementing of autosomal and Y-chromosomal STR markers in solving kinship-deficient cases involving father-daughter, mother-son, and sister-half-sister relationships, as well as those involving segregation and incest [1-3]. In 2018, Yu et al. [4] used X-STR to solve two cases, confirming that inclusion of more X-STRs may help solve complex kinship cases, which could not be resolved via autosomal STR markers. The number of loci and linkage group are important for the application of X-STR. At present in China, Argus X-12 is the most widely used multiplex amplification system. However, this system only encloses 12 X-STR loci located in 4 linkage groups, which is compounded by the fact that polymorphism of some loci in the Chinese population is less than satisfactory, limiting the efficacy of application. Furthermore, certain X-STR loci express strong linkage disequilibrium (LD) and segregate together as haplotypes [5-8]. Therefore, to develop a multiplex amplification system, which is more suitable for Chinese population is essential. DNATyper X19 Amplification Kit is self-developed by Institute of Forensic Science, Ministry of Public Security, aiming at demand of the cases, which is suitable for Chinese people. In this study, we investigated the forensic parameters from 374 unrelated Northern 
Chinese Han individuals, evaluating the value of DNATyper X19 kit in the forensic application.

\section{Materials and Methods}

\subsection{Samples}

Blood samples of 374 unrelated individuals (228 males and 146 females) were collected from North China. Blood samples were extracted using magnetic beads, and the genomic DNA was amplified with DNATyper X19 kit following manufacturer's recommendations.

\subsection{The General Information of DNATyper X19 kit}

The General information of all the loci in DNATyper X19 kit was shown in Table 1. 4-color fluorescent chemistry was used to enable multiplexing of 18 X-STRs and amelogenin, the amplicon size ranges no greater than $450 \mathrm{bp}$, which has high efficiency and reproducibility.

Table 1. General information of all the loci in DNATyper X19 kit

\begin{tabular}{|c|c|c|c|c|}
\hline Locus & Repeat motif & Chromosomal location & Allele area (bp) & Fluorescent type \\
\hline Amelogenin & NA & $\mathrm{Xp} 22.1-22.3$ & $100-110$ & FAM \\
\hline GATA31E08 & {$[\mathrm{AGAT}]_{\mathrm{n}}$} & $\mathrm{Xq} 27.1$ & $112-162$ & FAM \\
\hline DXS10079 & {$[\mathrm{AGAA}] \mathrm{AGAG}[\mathrm{AGAA}]_{\mathrm{n}}$} & $\mathrm{Xq} 12$ & $165-220$ & FAM \\
\hline DXS10103 & $\begin{array}{c}{[\mathrm{TAGA}] 2 \mathrm{CTGA}[\mathrm{CAGA}][\mathrm{TAGA}]_{\mathrm{n}}} \\
{[\mathrm{CAGA}][\mathrm{TAGA}]}\end{array}$ & $\mathrm{Xq} 26.2$ & $260-299$ & FAM \\
\hline DXS7132 & {$[\mathrm{TCTA}]_{\mathrm{n}}$} & $\mathrm{Xq12}$ & $304-351$ & FAM \\
\hline DXS9895 & $\begin{array}{c}{[\mathrm{AGAT}]_{\mathrm{n}} \mathrm{A}[\mathrm{AGAT}]_{\mathrm{m}}} \\
{[\mathrm{AGAT}]_{\mathrm{n}} \mathrm{AT}[\mathrm{AGAT}]_{\mathrm{m}}[\mathrm{AGAT}]_{3}}\end{array}$ & $\mathrm{Xp} 22.31$ & $370-410$ & FAM \\
\hline DXS7133 & {$[\mathrm{ATAG}]_{\mathrm{n}}$} & $\mathrm{Xq} 23$ & $94-145$ & HEX \\
\hline DXS7424 & {$[\mathrm{TAA}]_{\mathrm{n}}$} & $\mathrm{Xq} 22.1$ & $171-210$ & HEX \\
\hline DXS7423 & {$[\mathrm{TCCA}]_{\mathrm{n}}$} & $\mathrm{Xq} 28$ & $218-280$ & HEX \\
\hline DXS6789 & {$[$ TATC $][\text { TATG }]_{\mathrm{m}}[\text { TATC }]_{\mathrm{n}}$} & Xq21.33 & $310-383$ & HEX \\
\hline DXS9902 & {$[\mathrm{GATA}]_{\mathrm{n}}$} & Xp22.2 & $134-190$ & TAMRA \\
\hline DXS6810 & $\begin{array}{c}{[\mathrm{CTGT}]_{1}[\mathrm{CTAT}] 2[\mathrm{CTGT}]_{2}} \\
{[\mathrm{CTAT}]_{\mathrm{n}} \mathrm{CAT}[\mathrm{CTAT}]_{1}}\end{array}$ & Xp11.3 & $220-260$ & TAMRA \\
\hline DXS8377 & $\begin{array}{c}{[\mathrm{AGA}]_{\mathrm{x}}[\mathrm{GGAAGA}]_{\mathrm{y}}[\mathrm{AGA}]_{2}} \\
{[\mathrm{GGA}][\mathrm{AGA}]_{6}}\end{array}$ & $\mathrm{Xq} 28$ & $290-365$ & TAMRA \\
\hline DXS101 & {$[\mathrm{CTT}]_{\mathrm{m}}[\mathrm{ATT}]_{\mathrm{n}}$} & $\mathrm{Xq} 22.1$ & $375-430$ & TAMRA \\
\hline HPRTB & {$[\mathrm{AGAT}]_{\mathrm{n}}$} & $\mathrm{Xq} 26.2$ & $126-178$ & ROX \\
\hline DXS8378 & {$[\mathrm{CTAT}]_{\mathrm{n}}$} & $\mathrm{Xp} 22.31$ & $190-222$ & ROX \\
\hline DXS6797 & {$[\mathrm{ATCT}]_{\mathrm{n}}$} & $\mathrm{Xq} 22.3$ & $240-285$ & ROX \\
\hline DXS6804 & {$[\mathrm{TATC}]_{\mathrm{n}}$} & $\mathrm{Xq} 23$ & $293-340$ & ROX \\
\hline GATA165B12 & {$[\mathrm{AGAT}]_{\mathrm{n}}$} & $\mathrm{Xq} 24$ & $350-390$ & ROX \\
\hline
\end{tabular}

\subsection{DNA amplification}

DNA amplification was conducted with an Applied Biosystems ProFlex PCR System (Life Technologies, CA, USA). The reaction volume was $10 \mu \mathrm{L}$, containing $5 \mu \mathrm{L}$ of $2 \times$ PCR Master Mix, $2 \mu \mathrm{L}$ of $5 \times$ primer set, $1 \mu \mathrm{L}$ DNA, and $2 \mu \mathrm{L}$ of $\mathrm{ddH}_{2} \mathrm{O}$. The amplification program, based on the manufacturer's instructions, was as follows: $95^{\circ} \mathrm{C}$ for $15 \mathrm{~min}, 94^{\circ} \mathrm{C}$ for $30 \mathrm{~s}, 59^{\circ} \mathrm{C}$ for $45 \mathrm{~s}, 72^{\circ} \mathrm{C}$ for $1 \mathrm{~min}, 28 \mathrm{cycles}$, and $72^{\circ} \mathrm{C}$ for $60 \mathrm{~min}$.

\subsection{Electrophoresis and Analysis}


Electrophoresis was carried out by an Applied Biosystems 3500xl Genetic Analyzer (Life Technologies), GeneMapper ID-X v1.3 (Life Technologies) was used to analyze the DNA products. The sizes of allelic ladder and alleles were calculated via the supplied internal lane standard (Typer-500), which was modified with orange. An allele peak of 100 relative fluorescence units (RFU) was set as the threshold for peak detection.

\subsection{Statistical Analysis and Quality Control}

After the statistical analysis of all the genotyping, we obtained the heterozygosity $(\mathrm{H})$, matching probability (MP), polymorphism information content (PIC), discrimination power (DP) and power of exclusion (PE) of the 18 X-STR loci, which were calculated by the described methods [11], combined discrimination power (CDP) and combined mean exclusion chance (CMEC) were also calculated [12-15]. All DNA polymorphism analyses were conducted according to ISFG recommendations, as described by Schneider, et al. [16].

\section{Results and Discussion}

\section{Population Analysis}

Full profiles were obtained from the 374 samples, the profiles of 9947A and ladder were shown in Fig. 1 and Fig.2.

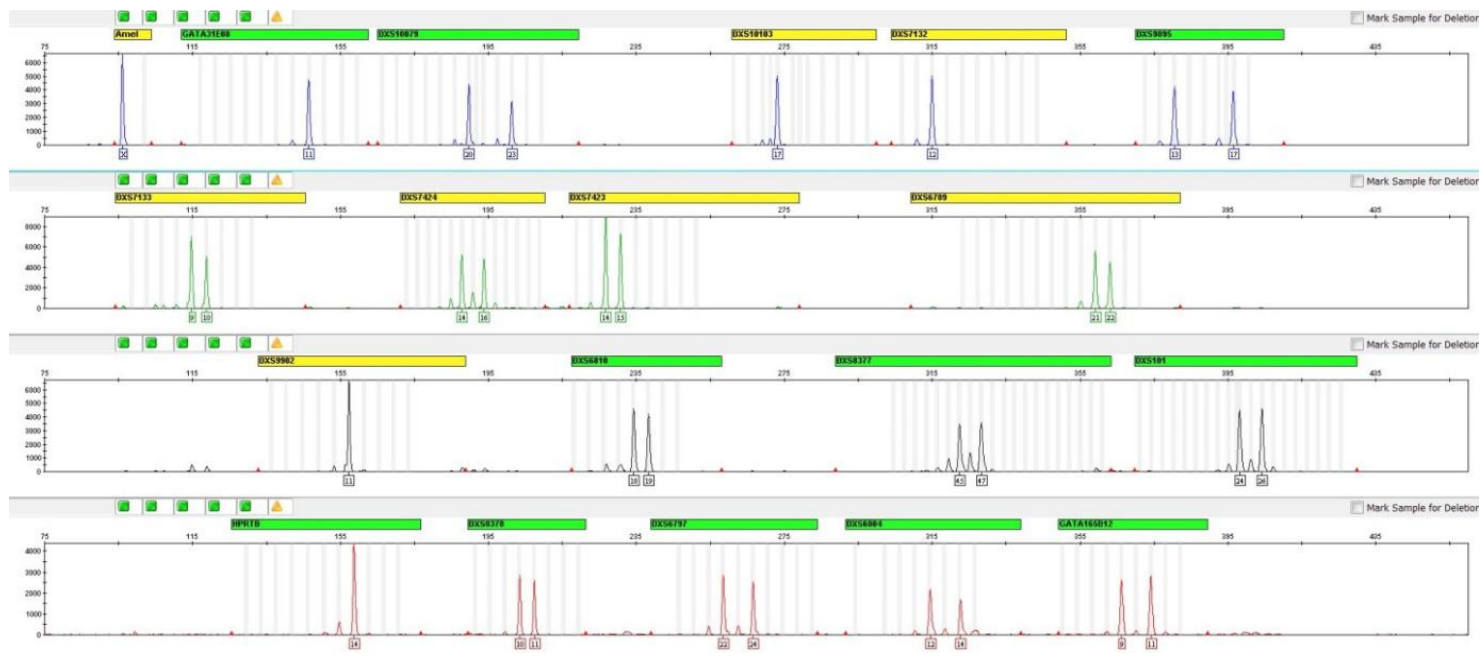

Fig. 1 Genotype profile of control 9947A at $1 \mathrm{ng}$

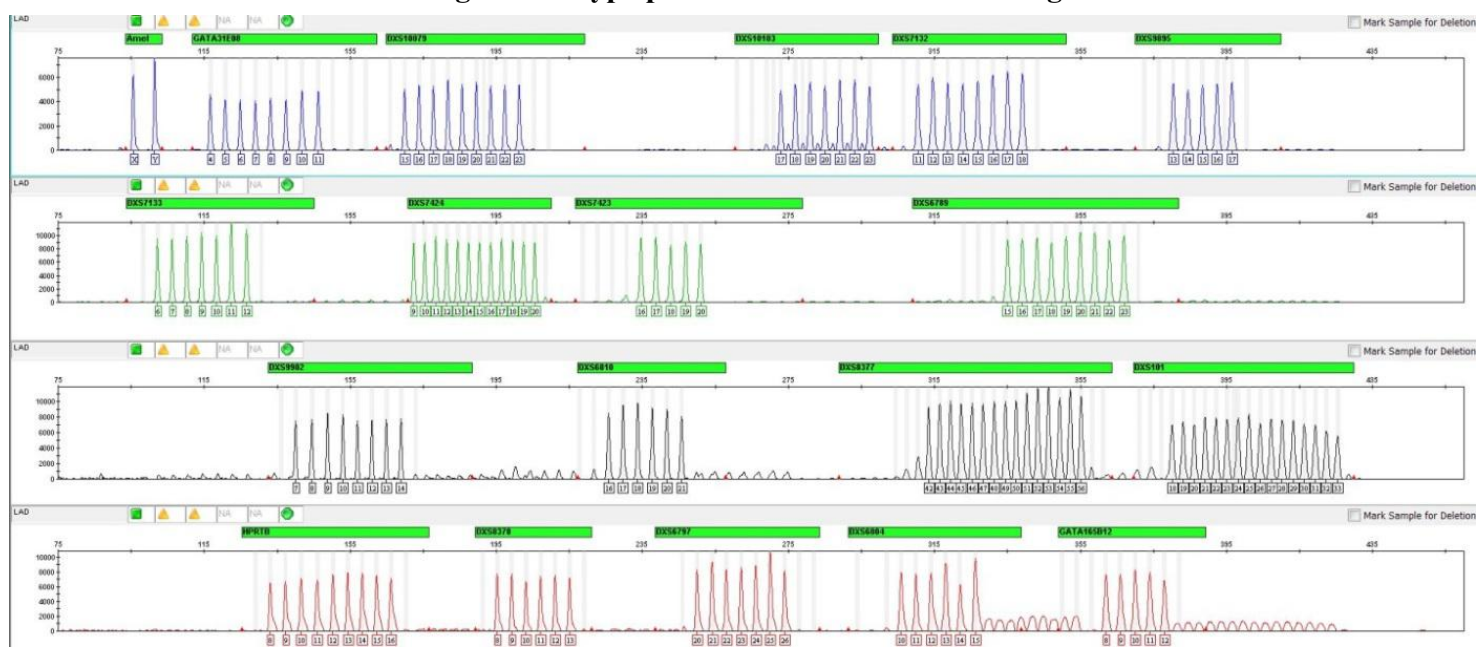

Fig. 2 Allelic ladder presenting its profile through a 3500xl genetic analyzer

The frequencies of the 18 X-STR loci were obtained from the genotyping of 374 unrelated Northern Chinese 
Han individuals (228 males, 146 females). Haplotypes of the 18 X-STR were calculated by simple counting based on the 228 typed male samples, a total of 228 haplotypes having been detected, the diversity is $100 \%$. The p-values for Hardy-Weinberg equilibrium based on 146 female samples were calculated with Powerstats software (shown in Table 2), linkage disequilibrium between 18 X-STR loci based on 228 male samples were calculated with Arlequin Ver3.5 software (shown in Table 3). No markers departed from Hardy-Weinberg equilibrium after applying Bonferroni's correction for multiple testing ( $\mathrm{p}=0.05 / 18)$, GATA31E08-DXS6797, DXS8377-DXS10079 were in linkage disequilibrium (LD) in this study ( $\mathrm{p}=0.05 / 153)$.

Table 2. The p-values for Hardy-Weinberg equilibrium at 18 X-STR loci (n=146)

\begin{tabular}{cll}
\hline Locus & P-values \\
\hline DXS101 & 0.04804 \\
DXS6789 & 0.96299 \\
DXS6797 & 0.49029 \\
DXS6804 & 0.90946 \\
DXS6810 & 0.51453 \\
DXS7133 & 0.64454 \\
DXS7423 & 0.24846 \\
DXS7424 & 0.42443 \\
DXS8377 & 0.42875 \\
DXS8378 & 0.13061 \\
DXS9895 & 0.85857 \\
DXS9902 & 0.67473 \\
DXS10079 & 0.90895 \\
DXS10103 & 0.13744 \\
GATA31E08 & 0.19116 \\
GATA165B12 & 0.15229 \\
HPRTB & 0.83427 \\
\hline
\end{tabular}

Table 3. The p-values for linkage disequilibrium between $18 \mathrm{X}$-STR loci $(\mathrm{n}=\mathbf{2 2 8})$

\begin{tabular}{|c|c|c|c|c|c|c|c|c|c|c|c|c|c|c|c|c|c|c|}
\hline & DXS101 & DXS6789 & DXS6797 & DXS6804 & DXS6810 & DXS7132 & DXS7133 & DXS7423 & DXS7424 & DXS8377 & DXS8378 & DXS9895 & DXS9902 & DXS10079 & DXS10103 & GATA31E08 & GATA165B12 & HPRTB \\
\hline \multicolumn{19}{|l|}{ DXS101 } \\
\hline DXS6789 & 0.9998 & & & & & & & & & & & & & & & & & \\
\hline DXS6797 & 0.9377 & 0.7254 & & & & & & & & & & & & & & & & \\
\hline DXS6804 & 0.2594 & 0.5507 & 0.9386 & & & & & & & & & & & & & & & \\
\hline DXS6810 & 0.8498 & 0.8289 & 0.5212 & 0.4417 & & & & & & & & & & & & & & \\
\hline DXS7132 & 0.8124 & 0.3224 & 0.0393 & 0.4267 & 0.6431 & & & & & & & & & & & & & \\
\hline DXS7133 & 0.2347 & 0.2755 & 0.4081 & 0.9279 & 0.7466 & 0.3564 & & & & & & & & & & & & \\
\hline DXS7423 & 0.1701 & 0.1876 & 0.4627 & 0.1196 & 0.8981 & 0.8225 & 0.2412 & & & & & & & & & & & \\
\hline DXS7424 & 0.8721 & 0.1664 & 0.9246 & 0.8583 & 0.4799 & 0.9702 & 0.8365 & 0.6100 & & & & & & & & & & \\
\hline DXS8377 & 0.1035 & 0.3967 & 0.8950 & 0.3325 & 0.5884 & 0.1519 & 0.9035 & 0.9754 & 0.6149 & & & & & & & & & \\
\hline DXS8378 & 1.0000 & 0.0608 & 0.9096 & 0.0100 & 0.7058 & 0.4910 & 0.0331 & 0.9739 & 0.5611 & 0.6527 & & & & & & & & \\
\hline DXS9895 & 0.3619 & 0.2747 & 0.2634 & 0.7863 & 0.3454 & 0.4823 & 0.5247 & 0.4235 & 0.2064 & 0.3999 & 0.8779 & & & & & & & \\
\hline DXS9902 & \begin{tabular}{|l|l}
0.9487 \\
\end{tabular} & 0.0914 & 0.3254 & 0.8829 & 0.7920 & 0.7760 & 0.4098 & 0.1479 & 0.8825 & 0.5665 & 0.5857 & 0.7097 & & & & & & \\
\hline DXS10079 & 0.3385 & 0.6204 & 0.8223 & 0.6169 & 0.8345 & 0.5341 & 0.6104 & 0.0654 & 0.2902 & 0.0000 & 0.9470 & 0.1076 & 0.8083 & & & & & \\
\hline DXS10103 & 0.0606 & 0.8862 & 0.0874 & 0.4245 & 0.1449 & 0.8203 & 0.6396 & 0.9854 & 0.9753 & 0.6449 & 0.0380 & 0.6596 & 0.1663 & 0.0986 & & & & \\
\hline GATA31E08 & 0.3257 & 0.8908 & 0.0000 & 0.1844 & 0.8604 & 0.7470 & 0.5989 & 0.8326 & 0.7970 & 0.4248 & 0.9480 & 0.5993 & 0.1958 & 0.6734 & 0.0231 & & & \\
\hline GATA165B12 & 0.5171 & 0.6296 & 0.0821 & 0.7543 & 0.4312 & 0.2701 & 0.9251 & 0.6589 & 0.1526 & 0.3029 & 0.7605 & 0.5742 & 0.8949 & 0.0199 & 0.3544 & 0.5614 & & \\
\hline HPRTB & 0.6659 & 0.8606 & 0.9338 & 0.1062 & 0.9130 & 0.5561 & 0.5292 & 0.4444 & 0.8922 & 0.9412 & 0.1248 & 0.8383 & 0.8602 & 0.3833 & 0.3673 & 0.8206 & 0.1281 & \\
\hline
\end{tabular}

A total of 140 alleles were detected from the 18 X-STR loci among the 374 individuals. There was no 
significant difference in the allele frequencies between male and female populations (shown in Table 4), $\mathrm{p}=0.05$. The genetic parameters of each locus (shown in Table 5) were calculated via the frequencies of the $18 \mathrm{X}$-STR loci. The combined discrimination power was 0.99999993 in male and 0.999999992 in female. The combined mean exclusion chance was 0.999993 in duo case $\left(\mathrm{CMEC}_{\text {duo }}\right)$ and 0.999999994 in trio case $\left(\mathrm{CMEC}_{\text {trio }}\right)$.

Table 4. The p-values of allele frequencies between male and female populations

\begin{tabular}{cc}
\hline Locus & P-values \\
\hline GATA31E08 & 0.18086 \\
DXS10079 & 0.68984 \\
DXS10103 & 0.88359 \\
DXS7132 & 0.90314 \\
DXS9895 & 0.8784 \\
DXS7133 & 0.61254 \\
DXS7424 & 0.82461 \\
DXS7423 & 0.7241 \\
DXS6789 & 0.5983 \\
DXS9902 & 0.92753 \\
DXS6810 & 0.12524 \\
DXS8377 & 0.40135 \\
DXS101 & 0.25558 \\
HPRTB & 0.24508 \\
DXS8378 & 0.71064 \\
DXS6797 & 0.71715 \\
DXS6804 & 0.85937 \\
GATA65B12 & 0.97006 \\
\hline
\end{tabular}

Table 5. Forensic parameters of 18 X-STR loci $(n=374)$

\begin{tabular}{|c|c|c|c|c|c|c|c|}
\hline \multirow{2}{*}{ Locus } & \multirow{2}{*}{$(\mathrm{H})$} & \multirow{2}{*}{ (MP) } & \multicolumn{2}{|c|}{ (DP) } & \multirow{2}{*}{ (PIC) } & \multicolumn{2}{|c|}{$(\mathrm{PE})$} \\
\hline & & & male & female & & trio case & duo case \\
\hline GATA31E08 & 0.76 & 0.185 & 0.757161 & 0.905909655 & 0.76 & 0.72204 & 0.58695457 \\
\hline DXS10079 & 0.783 & 0.162 & 0.77980302 & 0.921728769 & 0.783 & 0.75002 & 0.62082276 \\
\hline DXS10103 & 0.764 & 0.177 & 0.76089258 & 0.90496499 & 0.764 & 0.72303 & 0.58799948 \\
\hline DXS7132 & 0.754 & 0.187 & 0.7514365 & 0.897400963 & 0.754 & 0.71062 & 0.57309016 \\
\hline DXS9895 & 0.749 & 0.189 & 0.74643626 & 0.89078334 & 0.749 & 0.70151 & 0.56195359 \\
\hline DXS7133 & 0.434 & 0.508 & 0.43264341 & 0.63232965 & 0.434 & 0.38687 & 0.25287645 \\
\hline DXS7424 & 0.728 & 0.212 & 0.7252005 & 0.882285889 & 0.728 & 0.683 & 0.54324801 \\
\hline DXS7423 & 0.534 & 0.384 & 0.53262384 & 0.702922892 & 0.534 & 0.45399 & 0.31401303 \\
\hline DXS6789 & 0.83 & 0.121 & 0.82710646 & 0.947427424 & 0.83 & 0.80443 & 0.68848312 \\
\hline DXS9902 & 0.636 & 0.28 & 0.63368013 & 0.792919793 & 0.636 & 0.56079 & 0.41478505 \\
\hline DXS6810 & 0.604 & 0.315 & 0.60186317 & 0.774084505 & 0.604 & 0.53446 & 0.38752445 \\
\hline DXS8377 & 0.908 & 0.063 & 0.90448188 & 0.983060916 & 0.908 & 0.89667 & 0.81972967 \\
\hline DXS101 & 0.818 & 0.139 & 0.81482591 & 0.94118208 & 0.818 & 0.7903 & 0.67065759 \\
\hline HPRTB & 0.727 & 0.202 & 0.7249132 & 0.877935092 & 0.727 & 0.67852 & 0.53678585 \\
\hline DXS8378 & 0.586 & 0.335 & 0.58414226 & 0.76318956 & 0.586 & 0.52027 & 0.37440053 \\
\hline DXS6797 & 0.722 & 0.209 & 0.719265 & 0.874585879 & 0.722 & 0.67266 & 0.53051556 \\
\hline DXS6804 & 0.753 & 0.18 & 0.7499022 & 0.896046161 & 0.753 & 0.7085 & 0.57000007 \\
\hline GATA165B12 & 0.589 & 0.334 & 0.58652012 & 0.775672078 & 0.589 & 0.53316 & 0.38458674 \\
\hline
\end{tabular}




\section{Conclusion}

This study demonstrated that the combined mean exclusion chance of DNATyper X19 kit is high enough for complex paternity cases, there is no significant difference compared with other systems [17-21]. Therefore, the frequencies and parameters of the $18 \mathrm{X}$-STR loci in this study can be used in the casework.

\section{Funds}

Technology research program of Ministry of Public Security (No. 2016JSYJC14); Fundamental research fund of China Institute of Forensic Science, Ministry of Public Security (No. 2018JB024).

\section{References}

[1] Becker D, Rodig H, Augustin C, et al. (2008). Population genetic evaluation of eight X-chromosomal short tandem repeat loci using Mentype Argus X-8 PCR amplification kit. Forensic Science International: Genetics, 2(1): 69-74.

[2] Hatsch D, Keyser C, Hienne R, et al. (2007). Resolving paternity relationships using X-chromosome STRs and Bayesian networks. Journal of Forensic Sciences, 52(4): 895-897.

[3] Szibor R, Krawczak M, Hering S, et al. (2003). Use of X linked markers for forensic purposes. International Journal of Legal Medicine, 117(2): 67-74.

[4] Yu Z L, Sun J, Ding G S, et al. (2018). The application of X-STR: two case reports. Journal of Pharmacy and Pharmacology, 6(9): 859-862.

[5] Hundertmark T, Hering S, Edelmann J, et al. (2008). The STR cluster DXS10148-DXS8378-DXS10135 provides a powerful tool for X-chromosomal haplotyping at Xp22. International Journal of Legal Medicine, 122(6): 489-492.

[6] Edelmann J, Hering S, Augustin C, et al. (2008). Haplotypes and haplotype stability within a $126.6 \mathrm{~kb}$ region at Xq28. Forensic Science International: Genetics, 1(1): 554-556.

[7] Edelmann J, Hering S, Augustin C, et al. (2009). Chromosome X centromere region - haplotype frequencies for different populations. Forensic Science International: Genetics, 2(1): 398-399.

[8] Ferreira Da Silva I H, Barbosa A G, Azavedo D A, et al. (2010). An X-chromosome penta-plex in two linkage groups: haplotype data in Alagoas and Rio de Janeiro populations from Brazil. Forensic Science International: Genetics, 4(4): 95-100.

[9] DNA Advisory Board. Quality assurance standards DNA databasing laboratories. (2008). Forensic Science Communications, 10. https://www2.fbi.gov/hq/lab/fsc/backissu/oct2008/standards/2008_10_standards01a.htm.

[10] SWGDAM Executive Board. (2016) SWGDAM Validation Guidelines for DNA Analysis Methods, posting on www.swgdam.org [12-05]. https://docs.wixstatic.com/ugd/4344b0_813b241e8944497e99b9c45b163b76bd.pdf.

[11] Tereba A. (1999). Tools for analysis of population statistics. Profiles in DNA, USA: Gene-Press, 2(3): 14-16.

[12] Ge Jy, Eisenberg A, Budowle B. (2012). Developing criteria and data to determine best options for expanding the core CODIS loci. Investigative Genetics, 3(1): 1.

[13] Weir B S. (2004). Matching and partially-matching DNA profiles. Journal of Forensic Sciences, 49(5): 1009-1014.

[14] Ge Jy, Chakraborly R, Eisenberg A J, et al. (2011). Comparisons of familial DNA database searching strategies. Journal of Forensic Sciences, 56(6): 1448-1456.

[15] Ge Jy, Budowle B, Chakraborly R.(2011). Choosing relatives for DNA identification of missing persons. Journal of Forensic Sciences, 56(s1): 23-28.

[16] Schneider P M. (2007). Scientific standards for studies in forensic genetics. Forensic Science International, 165(2): $238-243$.

[17] Tomas C, Pereira V, Morling N. (2012). Analysis of 12 X-STRs in Greenlanders, Danes and Somalis using Argus X-12. International Journal of Legal Medicine, 126(1): 121-128.

[18] Zhang S, Zhao S, Zhu R, et al. (2012). Genetic polymorphisms of 12 X-STR for forensic purposes in Shanghai Han population from China. Molecular Biology Reports, 39(5): 5705-5707.

[19] Samejima M, Nakamura Y, Nambiar P, et al. (2012). Genetic study of 12 X-STRs in Malay population living in and around Kuala Lumpur using Investigator Argus X-12 kit. International Journal of Legal Medicine, 126(4): 677-683.

[20] Tillmar A O. (2012). Population genetic analysis of 12 X-STRs in Swedish population. Forensic Science International: Genetics, 6(2): 80-81.

[21] Shin S H, Yu J S, Park S W, et al. (2005). Genetic analysis of 18 X-linked short tandem repeat markers in Korean population. Forensic science international, 147(1): 35-41. 